\title{
Patterns of Traumatic Spinal Injuries in the Developing World: A Five-Year Longitudinal Review
}

\author{
Rishi M. Kanna, Sreeharsha Peddireddy, Ajoy P. Shetty, Shanmuganathan Rajasekaran \\ Department of Orthopaedics and Spine Surgery, Ganga Hospital, Coimbatore, India
}

\begin{abstract}
Study Design: Retrospective review.
Purpose: A 5-year longitudinal study documenting and comparing patterns of traumatic spinal injuries (TSIs) observed in developing countries.

Overview of Literature: Current knowledge of the patterns and epidemiology of TSI are based on evidence from developed countries and there is a lack of data from developing countries to enable a comparison of information to formulate healthcare policies.

Methods: A review of case records of all patients treated at a tertiary level trauma center over a 5-year period (2015-2019) was performed. Epidemiological, clinical, and radiological data were analyzed.

Results: The incidence of spinal trauma was $6.2 \%(2,065 / 33,072)$ among all trauma patients. Among these 2,065 patients, the mean age was $43.4 \pm 16.3$ years and $77.3 \%(n=1,596)$ were aged $21-60$ years. The major cause of injury was falls $(52.1 \%, n=1,069)$ and $49.8 \%$ were high-energy falls (>10 feet [ $=3.048 \mathrm{~m}])$. In patients with TSI due to falls, injuries occurred at the workplace $(\mathrm{n}=376)$, home $(n=309)$, trees ( $n=151)$, wells $(n=77)$, and electric poles ( $n=57)$. Road traffic accidents contributed to $42 \%$ ( $n=862)$ of TSIs and predominantly affected motorcyclists $(52 \%, n=467)$. Around half $(53.5 \%, n=1,005)$ of all patients were in the lower socioeconomic strata. The most common injury level was thoracic region $(37.2 \%, n=769)$. Spinal cord injury (SCI) occurred in $49 \%(n=1,011)$ of patients and $49.7 \%(n=1,028)$ had injuries associated with other organs.

Conclusions: Our study indicated different demographic patterns and epidemiological features of TSI compared with the Western literature, including a preponderance of young male patients, falls from heights, motorcycle accidents, and a larger percentage of SCI. The high number of falls at workplace indicates a lack of knowledge among the public and policy makers about safety measures.
\end{abstract}

Keywords: Spine; Trauma; Spinal injuries; Spinal cord injuries; Developing countries

\section{Introduction}

The calculated global incidence of traumatic spinal injuries (TSIs) is 10.5 cases per 100,000 persons, which is approximately 79,000 cases per year [1]. The costs associ- ated with pain alleviation, disability, social dependence, and health care are high, even in developed countries [2]. Previous studies on the epidemiology of TSI in developed countries demonstrated that proper documentation and analysis of the available data helped to formulate protocols

Received Jul 26, 2021; Revised Sep 10, 2021; Accepted Oct 5, 2021

Corresponding author: Rishi M. Kanna

Department of Orthopaedics and Spine Surgery, Ganga Hospital, Coimbatore, Tamil Nadu, India

Tel: +91-9486600798, Fax: +91-4222440798, E-mail: rishiortho@gmail.com 
useful for the prevention of spinal trauma and improving patient handling during transport and early care in the emergency room [3]. Oliver et al. [4] documented the changing trends in spinal injuries over a 13 -year period and reported that the incidence of cord injuries due to motor vehicle accidents (MVAs) markedly decreased during the study period and identified plausible reasons for this, including improvements in road traffic safety, public health campaigns, automobile safety legislation, and strict implementation of rules.

The literature surrounding TSIs is restricted to developed countries that have existing robust healthcare systems in place, and have focused predominantly on spinal cord injuries and selective spinal regions, such as cervical spinal injuries [1]. Chiu et al. [5] reviewed global epidemiological studies of TSIs over a 20 -year period and noted gross differences in the incidence, mortality rate, patients' age, sex, causes, and severity of injury between developed and developing countries. For example, trauma due to MVAs and falls were the most common modes of spinal injury and fractures worldwide $[1,5]$. Injuries due to adventure sports, violence, and gunshot were not uncommon in studies in the Western literature, whereas these were absent in developing regions of the world. In developing countries, the incidence of TSI due to falls could be higher than that of MVAs since injuries sustained due to falling from trees and electric poles, construction sites, industries, and falling into wells are common occurrences. Evidently, region-specific epidemiological data on TSI is important since injuries and outcomes are often determined by civil infrastructure, transport systems, strength of health care emergency services and the socioeconomic fabric of the population. Globally, India has the highest incidence of road traffic accidents (RTAs), with a predicted incidence of 55 accidents and 17 deaths per hour [6].

It is intuitive that the information from studies originating from developed countries could be of limited use in planning patient management and health care policy decisions of countries like India, where the number of studies performed to date on the incidence of spinal trauma, causation, and risk factors are too few and inadequate to draw reliable conclusions. One recent epidemiological studied examined only 92 patients with thoracolumbar spine fractures, whereas another multicenter study involving 14 centers across India (2017) only evaluated 192 spine trauma patients to examine the basic epidemiology of spinal injuries $[7,8]$.
Hence, an understanding of the incidence and detailed epidemiology of TSI on a large scale is essential and critical. Such studies would be beneficial to formulate protocols and policies in the management of spinal trauma.

\section{Materials and Methods}

\section{Study design}

This was a retrospective study of consecutive series of spinal trauma patients that were treated between January 2015 and December 2019 Ganga Medical Centre and Hospitals (GMCH), Coimbatore, India. The study was approved by the Institutional Review Board of GMCH (reference no., IRB-GMCH-17.08.19). The requirement for informed consent from individual patients was omitted because of the retrospective design of this study. All patients admitted for spinal injury at the institution were included in the study and patients with pathological fractures were excluded. The details of the patients (Table 1), including age, sex, occupation, time of injury, mode of injury, place of injury, time of hospitalization, systolic blood pressure on arrival, comorbidities, severity of spinal cord injury (SCI), associated axial and appendicular injuries, treatment modality used for spinal injury, medical insurance coverage, patients giving up treatment, and mortality were collected from patient records. Details about the level of spinal injury, number of levels injured, and fracture morphology were collected from the picture archiving and communication system.

\section{Statistical analysis}

Obtained data were entered into a spreadsheet and analysis was performed using IBM SPSS ver. 21.0 (IBM Corp., Armonk, NY, USA). Categorical variables were presented a numbers and percentages and continuous variables were presented as mean \pm standard deviation and median. Quantitative variables were compared using Mann-Whitney test between the two groups. Qualitative variables were compared using chi-square test and Fisher's exact test. All $p$-values $<0.05$ were considered statistically significant.

\section{Results}

The incidence of TSI among all trauma patients treated 
Table 1. Demographics of patients afflicted by traumatic spinal injuries

\begin{tabular}{|c|c|}
\hline Characteristic & Value \\
\hline Mean age (yr) & $43.3 \pm 16.34$ \\
\hline \multicolumn{2}{|l|}{ Sex } \\
\hline Male & 1,694 (82.03) \\
\hline Female & $371(17.97)$ \\
\hline \multicolumn{2}{|l|}{ Occupation } \\
\hline Unskilled manual labor & $398(19.27)$ \\
\hline Semiskilled labor & $30(1.45)$ \\
\hline Technical manual worker & $115(5.56)$ \\
\hline Agriculture & $438(21.21)$ \\
\hline Business & $100(4.84)$ \\
\hline Civil engineer & $6(0.29)$ \\
\hline Driver & $124(6.00)$ \\
\hline Electrician & $58(2.80)$ \\
\hline Home maker & $167(8.08)$ \\
\hline Military service & $6(0.29)$ \\
\hline Police & $15(0.72)$ \\
\hline Retired & $55(2.66)$ \\
\hline Sedentary worker & $201(9.73)$ \\
\hline Student & $184(8.91)$ \\
\hline Unemployed & $12(0.58)$ \\
\hline Unknown & $156(7.55)$ \\
\hline \multicolumn{2}{|l|}{ Mode of injury } \\
\hline Fall from height & $1,069(52.10)$ \\
\hline$>30 \mathrm{ft}$ height $(\%)$ & 9.17 \\
\hline 10-30 ft height (\%) & 40.60 \\
\hline$<10 \mathrm{ft}$ height $(\%)$ & 17.50 \\
\hline Slip and fall & 14.90 \\
\hline Unknown height & 18.20 \\
\hline Motor vehicle accident & $862(42.03)$ \\
\hline Motorcycle (\%) & 52.00 \\
\hline Car (\%) & 21.00 \\
\hline Heavy vehicle (bus/lorry) (\%) & 6.00 \\
\hline Pedestrians (\%) & 5.00 \\
\hline Others $(\%)$ & 16.00 \\
\hline Fall of heavy object & $93(4.50)$ \\
\hline Assault & $2(0.09)$ \\
\hline Bull-animal related injury & $24(1.16)$ \\
\hline Machine injury & $1(0.004)$ \\
\hline Penetrating injury & $1(0.004)$ \\
\hline Median transit time of transportation (hr) & 20 \\
\hline No. of total trauma cases & 33,072 \\
\hline
\end{tabular}

(Continued on next page)
Table 1. Continued

\begin{tabular}{lc}
\hline Characteristic & \multicolumn{1}{c}{ Value } \\
\hline No. of spinal column injury & $2,065(6.24$ of total trauma) \\
\hline No. of spinal cord injury & $1,011 / 2,065(48.96)$ \\
\hline Complete neuro-deficit & $681 / 2,065(32.97)$ \\
\hline Incomplete neuro-deficit & $327 / 2,065(15.83)$ \\
\hline Level of injury & \\
\hline \multicolumn{1}{c}{ Cervical } & $685(33.10)$ \\
\hline Thoracic & $769(37.20)$ \\
\hline \multicolumn{1}{c}{ Lumbar } & $530(25.60)$ \\
\hline Sacro-coccygeal & $81(3.90)$ \\
\hline
\end{tabular}

No. of levels injured

\begin{tabular}{|lr|}
\hline Single & $1,604(77.68)$ \\
\hline Double & $419(20.29)$ \\
\hline Triple & $38(1.84)$ \\
\hline Ouadruple & $4(0.19)$ \\
\hline A0 type of injury & \\
\hline A0 type A injury & $643(31.13)$ \\
\hline A0 type B injury & $679(32.88)$ \\
\hline A0 type C injury & $601(29.10)$ \\
\hline Traumatic disc osteophyte complex & $142(6.90)$ \\
\hline Other injuries in spinal trauma patients & \\
\hline Head injury & $427(20.67)$ \\
\hline Chest injury & $269(13.02)$ \\
\hline Facial injury & $80(3.87)$ \\
\hline Pelvic injury & $195(9.44)$ \\
\hline Abdominal injury & $56(2.77)$ \\
\hline
\end{tabular}

Comorbidities

\begin{tabular}{|cc|}
\hline Diabetes mellitus & $313(15.16)$ \\
\hline Hypertension & $223(10.80)$ \\
\hline Ischemic heart disease & $40(1.94)$ \\
\hline Hypothyroidism & $22(1.07)$ \\
\hline Bronchial asthma & $20(0.97)$ \\
\hline Osteoporosis & $37(1.79)$ \\
\hline Ankylosing spondylitis & $23(1.11)$ \\
\hline Diffuse idiopathic skeletal hyperostosis & $14(0.68)$ \\
\hline Surgical fixation total & $1,567(75.9)$ \\
\hline First 24 hr (\%) & 57.12 \\
\hline 24 to 48 hr (\%) & 26.90 \\
\hline After 48 hr (\%) & 16.59 \\
\hline Mean length of stay (day) & 8.87 \\
\hline Complications & \\
\hline Ventilatory support & $231(11.19)$ \\
\hline
\end{tabular}


Table 1. Continued

\begin{tabular}{|cc|}
\hline Characteristic & Value \\
\hline Tracheostomy & $197(9.54)$ \\
\hline Respiratory infection & $58(2.81)$ \\
\hline Urinary tract infection & $38(1.84)$ \\
\hline Pleural effusion & $20(0.97)$ \\
\hline Acute respiratory distress syndrome & $12(0.58)$ \\
\hline Wound dehiscence & $6(0.29)$ \\
\hline Wound infection & $7(0.34)$ \\
\hline Recurrent laryngeal nerve palsy & $3(0.15)$ \\
\hline Mortality & $15(0.73)$ \\
\hline
\end{tabular}

Values are presented as mean \pm standard deviation, number (\%), or number unless otherwise stated.

during the study period was $6.2 \%(2,065 / 33,072$ general trauma patients had spinal injuries in 5 years) (Table 1 ).

\section{Age and sex}

The majority $(82.03 \%, n=1,694)$ of patients were male and $17.97 \%(n=371)$ were female. The mean age of the patients was $43.4 \pm 16.3$ years, $6.63 \%$ were children and young adults, and $16.1 \%$ were aged $>60$ years. A total of $77.3 \%$ of spinal trauma patients were aged 20-60 years and the median transit time of transportation of the patient to hospital was 20 hours.

\section{Causes of spinal trauma}

The major causes of spine trauma were falls from a height (52.1\%, $n=1,069)$, MVAs $(42.0 \%, n=862)$, heavy objects landing on the spine $(4.5 \%, \mathrm{n}=93)$, and injuries caused by animals $(1.2 \%, \mathrm{n}=24)$. Only two cases of assault-related spinal trauma were documented.

Among spinal injuries caused by falls, the majority $(40.6 \%, n=434)$ were falls from a height of 10-30 feet (3.048-9.144 m). Falls included injuries incurred due to a fall from high structures and those into deep wells. In $17.5 \%(\mathrm{n}=187)$ of patients, the injury was sustained by falling from $<10$ feet $(3.048 \mathrm{~m})$ and in $9.2 \%(\mathrm{n}=98)$, it occurred from a fall from $>30$ feet $(9.144 \mathrm{~m})$. Furthermore, $14.9 \%(n=159)$ of TSIs due to falls were attributable to slip and fall on a flat surface and predominantly involved elderly patients (>60 years). In $18.2 \%$ of patients, specific information on the altitude could not be retrieved due to various factors, including poor general condition on arrival, multiple distracting injuries, low Glasgow Coma Scale, postinjury amnesia, and alcoholism.

In patients who sustained injuries due to falls, the places the injury occurred were the workplace/industry $(n=376)$, home $(n=309)$, from a tree $(n=151)$, into a well $(n=77)$, and from an electric pole $(n=57)$. None of the injured persons who sustained injuries due to a fall at work were wearing protective equipment or harnesses.

Among the 862 patients who sustained TSIs in MVAs, $52.2 \%(n=467)$ were riding motorcycles, $21.8 \%(n=188)$ were in a light motor vehicle (car), 6.3\% $(n=54)$ were traveling in a heavy motor vehicle (bus/lorry), and 5.2\% $(n=45)$ were pedestrians. Among the 467 motorcycle accidents, 287 (61.5\%) occurred due to a self-skid without any collision, $17.9 \%(n=84)$ were due to collisions with a car, $50(10.7 \%)$ were due to collisions with other motorcyclists, and $40(8.6 \%)$ involved collisions with heavy vehicles.

\section{Social strata of patients}

Around half of all injuries $(53.5 \%, n=1,005)$ occurred in people in lower socioeconomic strata who worked in agriculture, construction, and manual labor. Socially and economically dependent individuals, including students, homemakers, unemployed, and retired people were involved in $20.25 \%(n=418)$. On the other hand, $18.2 \%$ $(n=386)$ were involved in skilled and professional jobs and were the least affected.

\section{Characteristics of spinal injury}

The most common injured level of spine was thoracic (37.2\%, $\mathrm{n}=769)$, followed by cervical $(33.1 \%, \mathrm{n}=685)$, lumbar $(25.6 \%, \mathrm{n}=530)$, and sacrococcygeal $(3.9 \%, \mathrm{n}=81)$. Most patients $(77.7 \%, \mathrm{n}=1,604)$ had single-level injures, whereas $20.3 \%(n=419)$ had injuries at two levels. Injuries at three levels were seen in $1.9 \%(n=38)$ of the patients and four patients had injuries at four levels. Patients who sustained injuries due to falls had a higher incidence of multilevel fractures.

The AO classification of spine fractures was used to broadly classify the injuries. Based on this classification, 31.4\% $(n=643)$ of patients had A type injury, 33.1\% $(n=679)$ had B type injury, and 29.3\% $(n=601)$ had $C$ type injury. Traumatic disc osteophyte compressions of the cervical spine were seen in $6.2 \%(n=142)$ of patients.

SCI was present in 1,011 patients (49.0\%), among whom 
682 had complete motor neurological injury and 329 had incomplete deficits. Neurological deficit was graded using the American Spinal Injury Association (ASIA) scale and revealed that 571 (27.7\%) were ASIA-A, 110 (5.3\%) were ASIA-B, 155 (7.5\%) were ASIA-C, 172 (8.3\%) were ASIA$\mathrm{D}$, and intact neurology was observed in 1,057 (51.2\%).
Complete neurological deficit was observed in $40.86 \%$ of patients affected by a heavy object land on them.

Young patients ( $<10$ years old) had a higher incidence of thoracic injuries $(66.6 \%)$, whereas older patients $(>80$ years old) sustained more cervical injuries (50\%) $(p<0.05)$. Similarly, the youngest and oldest $(<10$ years old and $>80$
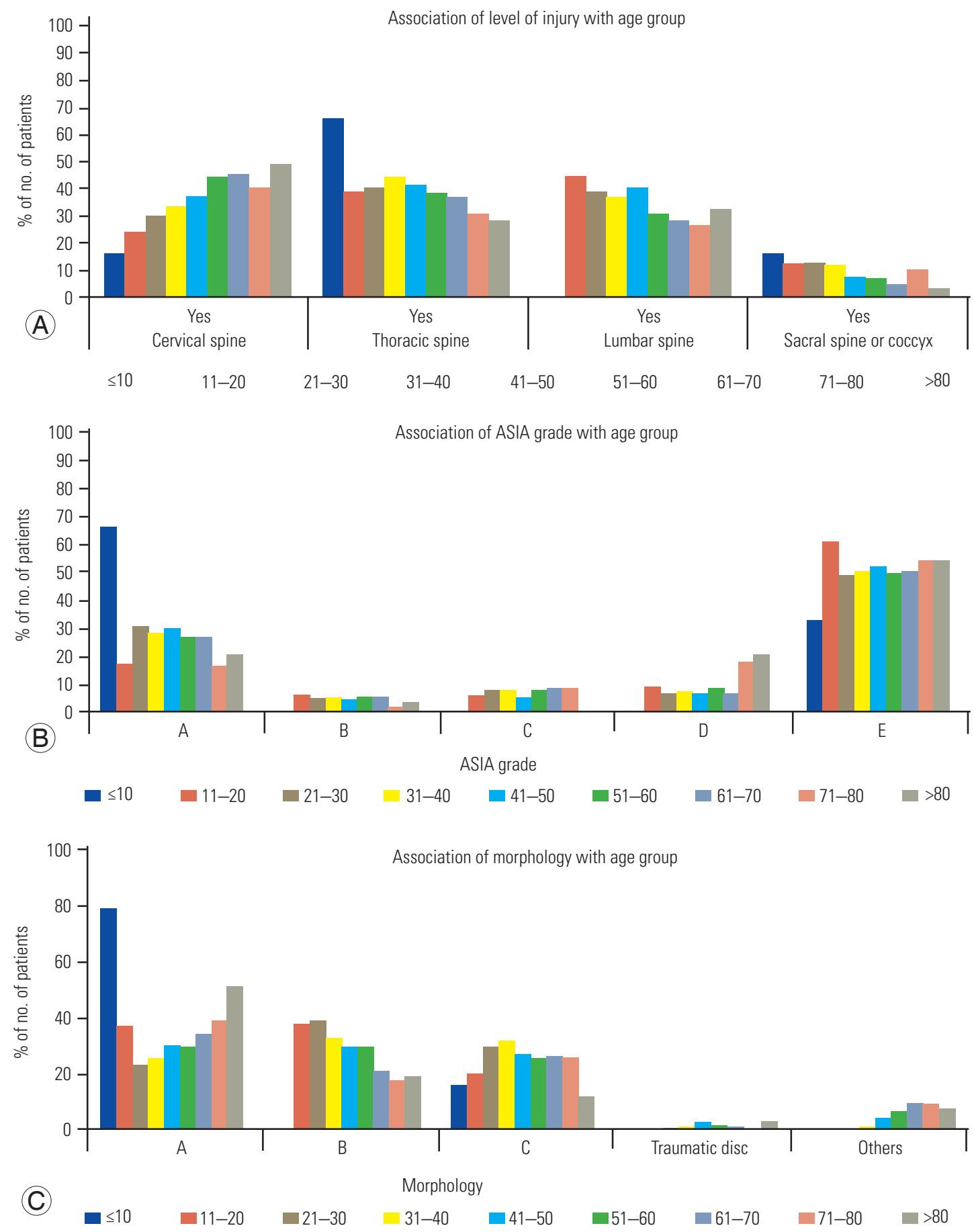

Fig. 1. Correlation between patients of different age groups with the level of spinal injury (A), American Spinal Injury Association (ASIA) neurological grade (B), and AO type of injury (C). 
years old) patients had a higher incidence of AO type A injuries $(p<0.0001)$ and all types, $\mathrm{A}-\mathrm{C}$, were uniformly distributed among the rest of the age groups. There were no specific patterns of neurological deficit in the different age groups, although ASIA-A injuries were higher among younger patients $(p<0.01)$ (Fig. 1).

A total of $75.9 \%(n=1,567)$ of patients underwent surgical fixation. The majority $(57.1 \%, \mathrm{n}=895)$ of these patients underwent surgical fixation of spine within the first 24 hours of admission, followed by $26.3 \%(n=412)$ during the next 24 hours and $16.6 \%(n=260)$ of patients underwent surgery 48 hours after admission.

\section{Associated injuries and comorbidities}

Around half of all patients $(49.7 \%, 1,028)$ had injuries associated with other organ systems, such as the head, chest, face, pelvis, and abdomen. Head injury was seen in 427 patients (20.7\%), chest injury in 269 (13.0\%), facial injury in 80 (3.9\%), pelvic injury in 195 (9.4\%), and abdominal injury in $56(2.7 \%)$. Comorbidities were documented in $601 \mathrm{pa}-$ tients (29.1\%), including diabetes mellitus (15.2\%, $\mathrm{n}=313)$, hypertension $(10.8 \%, \mathrm{n}=223)$, ischemic heart disease $(1.9 \%$, $\mathrm{n}=40)$, hypothyroidism $(1.1 \%, \mathrm{n}=22)$, respiratory diseases $(1.0 \%, \mathrm{n}=20)$, ankylosing spondylitis $(1.1 \%, \mathrm{n}=23)$, diffuse idiopathic skeletal hyperostosis $(0.7 \%, \mathrm{n}=14)$, and multiple comorbidities were noted in 54 patients.

\section{Hospital stay}

The mean and median lengths of stay in hospital after spinal injury were 8.9 days and 7 days, respectively. The mean length of stay reduced from $8.3 \pm 2.1$ to $6.2 \pm 1.7$ days during the 5 -year study period. Health insurance coverage was only $13.1 \%$ among all patients in the present study. A total of 15 out of 2,065 patients (0.7\%) died in hospital during treatment.

\section{Discussion}

The present study involved a large-scale longitudinal assessment of spinal injuries in the developing world and uniquely encompassed injuries to the whole spine as well as injuries to the vertebral column and spinal cord, which lack data. In a recent systematic review on global epidemiology of TSI, the authors highlighted the lack of information about TSI from developing and underdeveloped countries $[1,5]$. They noted that the majority of manuscripts included in the review included data from highincome countries (HICs, $\mathrm{n}=50$ ), whereas only six were from low-income countries (LICs). In their review, the incidence of TSI was higher in (LICs and middle-income countries [MICs]) (13.7 per 100,000 persons) compared with HICs (8.7 per 100,000 cases). The authors were concerned by this paradoxical situation of a lack of studies in regions of the world where the disease was prolific. Hence, the need for large-scale data on TSI and SCI from middle and LICs is clear and relevant, and our study provides important insight.

In the present study, the incidence of TSI among cases of general trauma was $6.2 \%$. The incidence of TSI varies in literature depending on the heterogeneity of the study population. Data from single-center studies provide similar results. Oliver et al. [4] analyzed TSIs over a 13-year period in a level 1 trauma center and observed that 5.8\% of all trauma patients suffered spinal fractures. Population-based studies provide an alternative perspective to disease incidence. The average incidence of hospitalized TSI was 26/100,000 in Northern Finland and a higher incidence of 64/100,000 in Canada, while Asian studies have shown incidences varying from 16-62 per 100,000 $[9,10]$.

In developing countries, the mechanism of injury causing TSI was different from that described in the Western literature. In the present study, the majority (52.1\%) of spinal trauma was due to falls from different heights, with the most common being from 10-30 feet (3.048-9.144 m). Such falls invariably occurred in construction site workers, industrial workers, tree climbers, and electric pole workers. This indicates the vulnerability of these workers to sustain injuries from such heights and a lack of knowledge about these accidents. The lack of availability of protective measures, such as harnesses and lanyards for these workers, is a serious social situation that warrants further investigation and remedial measures. Furthermore, there was a high incidence of TSI noted in low socioeconomic strata laborers in our study, with $53.5 \%(n=1,005)$ of TSIs occurring in people working in agriculture, construction, and manual labor.

Falls were the predominant mode of injury in recent studies; however, these were low falls $(<1 \mathrm{~m})$ sustained by elderly patients. In the present study, low falls were observed in $7.6 \%(n=159)$ of TSIs and predominantly occurred in people $>60$ years. Low fall was the most common etiology with $36 \%$ in a Finnish study, whereas low 
fall accounted for only $20 \%$ and $22 \%$ of injuries in studies based in Germany and China, respectively $[9,11,12]$. From a broader perspective, it is evident that studies quoting a higher incidence of TSIs due to low falls studied older populations and a lower incidence of unstable injuries, whereas studies documenting more TSIs due to high falls studied a younger population and high-velocity unstable injuries.

Most previous studies have reported RTAs to be the major cause of TSIs $[13,14]$. Studies by Oliver et al. [4], Pirouzmand [13], and Jackson et al. [14] reported MVAs as the major mechanism of spinal injuries at $54.7 \%, 66 \%$, and $45.6 \%$, respectively. However, improvements in road safety measures, civil infrastructure, and transport systems have contributed to a reduction in the incidence of TSIs due to RTAs in the Western world. In the present study, $42.1 \%$ of TSIs were related to MVAs. However, the majority of injuries were not sustained by car collisions. Interestingly, $52 \%$ of spinal trauma due to RTAs occurred in motorcyclists, which indicates the inherent danger of motorcycle riding in terms of sustaining a spinal injury. Further analysis indicated that $61.5 \%$ of TSIs in motorcyclists occurred due to a self-fall, indicating unsafe driving in this part of the world. Therefore, strict adherence to the rules of the road for driving safety could reduce this subset of trauma.

The mean age of the patients with TSI was $43.4 \pm 16.3$ years, which was comparable to other studies. Notably, $77.3 \%$ of spinal trauma patients were aged $20-60$ years, which is the social and economically productive age group of a society. This assumes a larger significance since we observed that SCI and neurological deficit was documented in $49 \%$ of patients.

In our study population, thoracic spinal injury was observed in $40.8 \%$, cervical spinal injury was seen in $37.4 \%$, lumbar spinal injury in $36.8 \%$, and $10.5 \%$ patients had sacrococcygeal injuries. The predominant location of injury depends on the selected population subset. Population studies that describe a higher number of low falls sustained in elderly population have documented a higher incidence of lumbar and lumbosacral fractures $[9,12,13]$. Studies focusing on SCIs have indicated a higher number of cervical spinal injuries [15]. The present study also revealed a high $(49.1 \%)$ incidence of spinal cord injuries, which was much higher than that reported in the literature. Apart from the higher incidence of high falls and motorcycle accidents, which are high-magnitude injuries that can cause vertebral translation, our center offers dedicated spinal rehabilitation services that could have led to this observation of a higher incidence of SCI. Injuries associated with other organ systems can affect the management and outcomes of patients with TSIs. The frequency of these injuries $(49.1 \%)$ was similar to those reported in other studies, which showed frequencies of 38\%-54\% $[12,16]$. The average transit time of 20 hours noted in our study was much higher than a study conducted in Canada, which reported 2.5 hours [13].

The present study has some limitations. First, this was a retrospective study conducted at a single specialty center for trauma and orthopedics. Second, data maintenance was based on human data entry. Third, less serious spinal injuries could have been treated at smaller centers and more seriously injured patients who died before reaching the hospital could have been missed in the analysis.

\section{Conclusions}

Our study indicates different demographic patterns and epidemiological features of TSIs in developing countries compared with those reported in the Western literature. A preponderance of young male patients, high falls, motorcycle accidents, and a larger percentage of SCI were distinct observations in this large-scale study. Furthermore, the high number of workplace injuries indicates a lack of knowledge about safety measures of protection. TSIs currently affect a higher number of patients in lower socioeconomic strata and could be a preventable injury if improvements in awareness and public educational measures can be implemented.

\section{Conflict of Interest}

No potential conflict of interest relevant to this article was reported.

\section{Acknowledgments}

The study was funded by the Ganga Orthopaedic Research and Education Foundation (GOREF).

\section{Author Contributions}

Rishi M. Kanna: conceptualisation, guidance, writing; Sreeharsha Peddireddy: data collection, entry; Ajoy P. 
Shetty: guidance in concepts, design of the work, drafting the work; and Shanmuganathan Rajasekaran: interpretation of data, revising it critically, final approval.

\section{References}

1. Kumar R, Lim J, Mekary RA, et al. Traumatic spinal injury: global epidemiology and worldwide volume. World Neurosurg 2018;113:e345-63.

2. Baaj AA, Uribe JS, Nichols TA, et al. Health care burden of cervical spine fractures in the United States: analysis of a nationwide database over a 10 -year period. J Neurosurg Spine 2010;13:61-6.

3. Burton JH, Dunn MG, Harmon NR, Hermanson TA, Bradshaw JR. A statewide, prehospital emergency medical service selective patient spine immobilization protocol. J Trauma 2006;61:161-7.

4. Oliver M, Inaba K, Tang A, et al. The changing epidemiology of spinal trauma: a 13-year review from a Level I trauma centre. Injury 2012;43:1296-300.

5. Chiu WT, Lin HC, Lam C, Chu SF, Chiang YH, Tsai SH. Review paper: epidemiology of traumatic spinal cord injury: comparisons between developed and developing countries. Asia Pac J Public Health 2010;22:9-18.

6. World Health Organization. Global status report on road safety 2018. Geneva: World Health Organization; 2018.

7. Khurjekar K, Hadgaonkar S, Kothari A, et al. Demographics of thoracolumbar fracture in Indian population presenting to a tertiary level trauma centre. Asian Spine J 2015;9:344-51.
8. Aleem IS, DeMarco D, Drew B, et al. The burden of spine fractures in India: a prospective multicenter study. Global Spine J 2017;7:325-33.

9. Niemi-Nikkola V, Saijets N, Ylipoussu H, et al. Traumatic spinal injuries in northern Finland. Spine (Phila Pa 1976) 2018;43:E45-51.

10. Hu R, Mustard CA, Burns C. Epidemiology of incident spinal fracture in a complete population. Spine (Phila Pa 1976) 1996;21:492-9.

11. Leucht P, Fischer K, Muhr G, Mueller EJ. Epidemiology of traumatic spine fractures. Injury 2009;40:16672.

12. Wang H, Zhang $Y$, Xiang Q, et al. Epidemiology of traumatic spinal fractures: experience from medical university-affiliated hospitals in Chongqing, China, 2001-2010. J Neurosurg Spine 2012;17:459-68.

13. Pirouzmand F. Epidemiological trends of spine and spinal cord injuries in the largest Canadian adult trauma center from 1986 to 2006. J Neurosurg Spine 2010;12:131-40.

14. Jackson AB, Dijkers M, Devivo MJ, Poczatek RB. A demographic profile of new traumatic spinal cord injuries: change and stability over 30 years. Arch Phys Med Rehabil 2004;85:1740-8.

15. Pickett GE, Campos-Benitez M, Keller JL, Duggal N. Epidemiology of traumatic spinal cord injury in Canada. Spine (Phila Pa 1976) 2006;31:799-805.

16. Hasler RM, Exadaktylos AK, Bouamra O, et al. Epidemiology and predictors of spinal injury in adult major trauma patients: European cohort study. Eur Spine J 2011;20:2174-80. 